\title{
Coefficient estimates for new subclasses of Ma-Minda bi-univalent functions
}

\author{
Huo Tang ${ }^{1,2^{*}}$, Guan-Tie Deng ${ }^{2}$ and Shu-Hai Li ${ }^{1}$ \\ Dedicated to Professor Hari M Srivastava
}

"Correspondence:

thth2009@163.com

${ }^{1}$ School of Mathematics and

Statistics, Chifeng University,

Chifeng, Inner Mongolia 024000,

China

2School of Mathematical Sciences, Beijing Normal University, Beijing, 100875, China

\begin{abstract}
In this paper, we introduce and investigate two new subclasses $H_{\sigma}^{\mu}(\lambda, \varphi)$ and $M_{\sigma}^{\gamma}(\lambda, \mu, \varphi)$ of Ma-Minda bi-univalent functions defined by using subordination in the open unit disk $D=\{z \in C:|z|<1\}$. For functions belonging to these new subclasses, we obtain estimates for the initial coefficients $\left|a_{2}\right|$ and $\left|a_{3}\right|$. The results presented in this paper would generalize those in related works of several earlier authors.

MSC: $30 C 45 ; 30 C 80$
\end{abstract}

Keywords: analytic and univalent functions; bi-univalent functions; coefficient estimates; subordination

\section{Introduction}

Let $C$ be a set of complex numbers and let $N=\{1,2,3, \ldots\}=N_{0} \backslash\{0\}$ be a set of positive integers. Let $A$ be a class of functions of the form

$$
f(z)=z+\sum_{n=2}^{\infty} a_{n} z^{n}
$$

which are analytic in the open unit disk $D=\{z \in C:|z|<1\}$. Also, let $S$ denote a subclass of all functions in $A$ which are univalent in $D$ (for details, see [1, 2]).

Since univalent functions are one-to-one, they are invertible and the inverse functions need not be defined on the entire unit disk $D$. However, the famous Koebe one-quarter theorem [1] ensures that the image of the unit disk $D$ under every function $f \in S$ contains a disk of radius $1 / 4$. Thus, every univalent function $f \in S$ has an inverse $f^{-1}$ satisfying

$$
f^{-1}(f(z))=z \quad(z \in D)
$$

and

$$
f\left(f^{-1}(w)\right)=w \quad\left(|w|<r_{0}(f) ; r_{0}(f) \geq \frac{1}{4}\right),
$$

where

$$
f^{-1}(w)=w-a_{2} w^{2}+\left(2 a_{2}^{2}-a_{3}\right) w^{3}-\left(5 a_{2}^{3}-5 a_{2} a_{3}+a_{4}\right) w^{4}+\cdots
$$

C 2013 Tang et al.; licensee Springer. This is an Open Access article distributed under the terms of the Creative Commons Attribution License (http://creativecommons.org/licenses/by/2.0), which permits unrestricted use, distribution, and reproduction in any medium, provided the original work is properly cited. 
A function $f \in A$ is said to be bi-univalent in $D$ if both $f$ and $f^{-1}$ are univalent in $D$. Let $\sigma$ denote the class of bi-univalent functions defined in the unit disk $D$. In 1967, Lewin [3] first introduced the class $\sigma$ of bi-univalent functions and showed that $\left|a_{2}\right| \leq 1.51$ for every $f \in \sigma$. Subsequently, Branan and Clunie [4] conjectured that $\left|a_{2}\right| \leq \sqrt{2}$ for $f \in \sigma$. Later, Netanyahu [5] proved that $\max _{f \in \sigma}\left|a_{2}\right|=3 / 4$. The coefficient estimate problem for each of $\left|a_{n}\right|(n \in N \backslash\{1,2\})$ is still an open problem.

Brannan and Taha [6] (see also [7]) introduced certain subclasses of a bi-univalent function class $\sigma$ similar to the familiar subclasses $S^{*}(\alpha)$ and $K(\alpha)$ of starlike and convex functions of order $\alpha(0<\alpha \leq 1)$, respectively (see [8]). Thus, following Brannan and Taha [6] (see also [7]), a function $f \in A$ is in the class $S_{\sigma}^{*}[\alpha]$ of strongly bi-starlike functions of or$\operatorname{der} \alpha(0<\alpha \leq 1)$ if both functions $f$ and $f^{-1}$ are strongly starlike functions of order $\alpha$. The classes $S_{\sigma}^{*}(\alpha)$ and $K_{\sigma}(\alpha)$ of bi-starlike functions of order $\alpha$ and bi-convex functions of order $\alpha$, corresponding (respectively) to the function classes $S^{*}(\alpha)$ and $K(\alpha)$, were also introduced analogously. For each of the function classes $S_{\sigma}^{*}(\alpha)$ and $K_{\sigma}(\alpha)$, they found nonsharp estimates on the first two Taylor-Maclaurin coefficients $\left|a_{2}\right|$ and $\left|a_{3}\right|$ (for details, see $[6,7])$.

An analytic function $f$ is subordinate to an analytic function $g$, written $f \prec g$, if there is an analytic function $w$ with $|w(z)| \leq|z|$ such that $f=(g(w))$. If $g$ is univalent, then $f \prec g$ if and only if $f(0)=g(0)$ and $f(D) \subseteq g(D)$. Ma and Minda [9] unified various subclasses of starlike and convex functions for which either of the quantities $z f^{\prime}(z) / f(z)$ or $1+z f^{\prime \prime}(z) / f^{\prime}(z)$ is subordinate to a more general superordinate function. For this purpose, they considered an analytic function $\varphi$ with positive real part in the unit disk $D, \varphi(0)=1, \varphi^{\prime}(0)>0$, and $\varphi$ maps $D$ onto a region starlike with respect to 1 and symmetric with respect to the real axis. The classes $S^{*}(\varphi)$ and $K(\varphi)$ of Ma-Minda starlike and Ma-Minda convex functions are respectively characterized by $z f^{\prime}(z) / f(z) \prec \varphi(z)$ or $1+z f^{\prime \prime}(z) / f^{\prime}(z) \prec \varphi(z)$. A function $f$ is bi-starlike of Ma-Minda type or bi-convex of Ma-Minda type if both $f$ and $f^{-1}$ are respectively Ma-Minda starlike or convex. These classes are denoted respectively by $S_{\sigma}^{*}(\varphi)$ and $K_{\sigma}(\varphi)$. Recently, Srivastava et al. [10], Frasin and Aouf [11] and Caglar et al. [12] introduced and investigated various subclasses of bi-univalent functions and found estimates on the coefficients $\left|a_{2}\right|$ and $\left|a_{3}\right|$ for functions in these classes. Very recently, Ali et al. [13], Kumar et al. [14], Srivastava et al. [15] and Xu et al.[16] unified and extended some related results in $[7,10-12,17]$ by generalizing their classes using subordination.

Motivated by Ali et al. [13] and Kumar et al. [14], we investigate the estimates for the initial coefficients $\left|a_{2}\right|$ and $\left|a_{3}\right|$ of bi-univalent functions of Ma-Minda type belonging to the classes $H_{\sigma}^{\mu}(\lambda, \varphi)$ and $M_{\sigma}^{\gamma}(\lambda, \mu, \varphi)$ defined in Section 2. Our results generalize several well-known results in [10-14] and these are also pointed out.

\section{Coefficient estimates}

Throughout this paper, we assume that $\varphi$ is an analytic univalent function with positive real part in $D, \varphi(D)$ is symmetric with respect to the real axis and starlike with respect to $\varphi(0)=1$, and $\varphi^{\prime}(0)>0$. Such a function has series expansion of the form

$$
\varphi(z)=1+B_{1} z+B_{2} z_{2}+B_{3} z_{3}+\cdots \quad\left(B_{1}>0\right) .
$$

With this assumption on $\varphi$, we now introduce the following subclasses of Ma-Minda biunivalent functions. 
Definition 2.1 A function $f \in \sigma$ given by (1.1) is said to be in the class $H_{\sigma}^{\mu}(\lambda, \varphi)$ if it satisfies

$$
(1-\lambda)\left(\frac{f(z)}{z}\right)^{\mu}+\lambda f^{\prime}(z)\left(\frac{f(z)}{z}\right)^{\mu-1} \prec \varphi(z) \quad(\lambda \geq 1, \mu \geq 1, z \in D)
$$

and

$$
(1-\lambda)\left(\frac{g(w)}{w}\right)^{\mu}+\lambda g^{\prime}(w)\left(\frac{g(w)}{z}\right)^{\mu-1} \prec \varphi(w) \quad(\lambda \geq 1, \mu \geq 1, w \in D)
$$

where the function $g$ is given by

$$
g(w)=f^{-1}(w)=w-a_{2} w^{2}+\left(2 a_{2}^{2}-a_{3}\right) w^{3}-\left(5 a_{2}^{3}-5 a_{2} a_{3}+a_{4}\right) w^{4}+\cdots .
$$

We note that, for suitable choices $\lambda, \mu$ and $\varphi$, the class $H_{\sigma}^{\mu}(\lambda, \varphi)$ reduces to the following known classes.

(1) $H_{\sigma}^{\mu}\left(\lambda,\left(\frac{1+z}{1-z}\right)^{\alpha}\right)=H_{\sigma}^{\mu}(\lambda, \alpha)(\lambda \geq 1,0<\alpha \leq 1, \mu \geq 0)$ (see Caglar et al. [12, Definition 2.1]);

(2) $H_{\sigma}^{\mu}\left(\lambda, \frac{1+(1-2 \beta) z}{1-z}\right)=H_{\sigma}^{\mu}(\lambda, \beta)(\lambda \geq 1,0 \leq \beta<1, \mu \geq 0)$ (see Caglar et al. [12, Definition 3.1]);

(3) $H_{\sigma}^{1}(\lambda, \varphi)=H_{\sigma}(\lambda, \varphi)(\lambda \geq 1)$ (see Kumar et al. [14, Definition 1.1]);

(4) $H_{\sigma}^{\mu}(1, \varphi)=H_{\sigma}^{\mu}(\varphi)(\mu \geq 0)$ (see Kumar et al. [14, Definition 2.1]);

(5) $H_{\sigma}^{1}(1, \varphi)=H_{\sigma}(\varphi)$ (see Ali et al. [13, p.345]);

(6) $H_{\sigma}^{1}\left(\lambda,\left(\frac{1+z}{1-z}\right)^{\alpha}\right)=H_{\sigma}(\lambda, \alpha)(\lambda \geq 1,0<\alpha \leq 1)$ (see Frasin and Aouf [11, Definition 2.1]);

(7) $H_{\sigma}^{1}\left(\lambda, \frac{1+(1-2 \beta) z}{1-z}\right)=H_{\sigma}(\lambda, \beta)(\lambda \geq 1,0 \leq \beta<1)$ (see Frasin and Aouf [11, Definition 3.1]);

(8) $H_{\sigma}^{1}\left(1,\left(\frac{1+z}{1-z}\right)^{\alpha}\right)=H_{\sigma}(\alpha)(0<\alpha \leq 1)$ (see Srivastava et al. [10, Definition 1]);

(9) $H_{\sigma}^{1}\left(1, \frac{1+(1-2 \beta) z}{1-z}\right)=H_{\sigma}(\beta)(0 \leq \beta<1)$ (see Srivastava et al. [10, Definition 2]).

For functions in the class $H_{\sigma}^{\mu}(\lambda, \varphi)$, the following estimates are obtained.

Theorem 2.1 Let the function $f$ given by (1.1) be in the class $H_{\sigma}^{\mu}(\lambda, \varphi), \lambda \geq 1$ and $\mu \geq 0$. Then

$$
\left|a_{2}\right| \leq \min \left\{\frac{B_{1}}{\lambda+\mu}, \sqrt{\frac{2\left(B_{1}+\left|B_{2}-B_{1}\right|\right)}{(1+\mu)(2 \lambda+\mu)}}\right\}
$$

and

$$
\left|a_{3}\right| \leq \begin{cases}\min \left\{\frac{B_{1}}{2 \lambda+\mu}+\frac{B_{1}^{2}}{(\lambda+\mu)^{2}}, \frac{2\left(B_{1}+\left|B_{2}-B_{1}\right|\right)}{(1+\mu)(2 \lambda+\mu)}\right\}, & 0 \leq \mu<1 \\ \frac{B_{1}}{2 \lambda+\mu}+\frac{2\left|B_{2}-B_{1}\right|}{(1+\mu)(2 \lambda+\mu)}, & \mu \geq 1\end{cases}
$$

Proof Since $f \in H_{\sigma}^{\mu}(\lambda, \varphi)$, there exist two analytic functions $u, v: D \rightarrow D$, with $u(0)=v(0)=$ 0 , such that

$$
(1-\lambda)\left(\frac{f(z)}{z}\right)^{\mu}+\lambda f^{\prime}(z)\left(\frac{f(z)}{z}\right)^{\mu-1}=\varphi(u(z))
$$


and

$$
(1-\lambda)\left(\frac{g(w)}{w}\right)^{\mu}+\lambda g^{\prime}(w)\left(\frac{g(w)}{z}\right)^{\mu-1}=\varphi(v(w))
$$

Define the functions $p$ and $q$ by

$$
\begin{aligned}
& p(z)=\frac{1+u(z)}{1-u(z)}=1+p_{1} z+p_{2} z^{2}+\cdots \quad \text { and } \\
& q(z)=\frac{1+v(z)}{1-v(z)}=1+q_{1} z+q_{2} z^{2}+\cdots
\end{aligned}
$$

or, equivalently,

$$
u(z)=\frac{p(z)-1}{p(z)+1}=\frac{1}{2}\left(p_{1} z+\left(p_{2}-\frac{p_{1}^{2}}{2}\right) z^{2}+\cdots\right)
$$

and

$$
v(z)=\frac{q(z)-1}{q(z)+1}=\frac{1}{2}\left(q_{1} z+\left(q_{2}-\frac{q_{1}^{2}}{2}\right) z^{2}+\cdots\right) .
$$

It is clear that $p$ and $q$ are analytic in $D$ and $p(0)=q(0)=1$. Since $u, v: D \rightarrow D$, the functions $p$ and $q$ have positive real part in $D$, and hence $\left|p_{i}\right| \leq 2$ and $\left|q_{i}\right| \leq 2(i=1,2, \ldots)$. By virtue of (2.7), (2.8), (2.10) and (2.11), we have

$$
(1-\lambda)\left(\frac{f(z)}{z}\right)^{\mu}+\lambda f^{\prime}(z)\left(\frac{f(z)}{z}\right)^{\mu-1}=\varphi\left(\frac{p(z)-1}{p(z)+1}\right)
$$

and

$$
(1-\lambda)\left(\frac{g(w)}{w}\right)^{\mu}+\lambda g^{\prime}(w)\left(\frac{g(w)}{z}\right)^{\mu-1}=\varphi\left(\frac{q(w)-1}{q(w)+1}\right) .
$$

Using (2.10), (2.11), together with (2.1), we easily obtain

$$
\varphi\left(\frac{p(z)-1}{p(z)+1}\right)=1+\frac{1}{2} B_{1} p_{1} z+\left(\frac{1}{2} B_{1}\left(p_{2}-\frac{1}{2} p_{1}^{2}\right)+\frac{1}{4} B_{2} p_{1}^{2}\right) z^{2}+\cdots
$$

and

$$
\varphi\left(\frac{q(w)-1}{q(w)+1}\right)=1+\frac{1}{2} B_{1} q_{1} w+\left(\frac{1}{2} B_{1}\left(q_{2}-\frac{1}{2} q_{1}^{2}\right)+\frac{1}{4} B_{2} q_{1}^{2}\right) w^{2}+\cdots
$$

Since $f \in \sigma$ has the Maclaurin series given by (1.1), a computation shows that its inverse $g=f^{-1}$ has the expansion given by (1.2). Also, since

$$
\begin{aligned}
& f^{\prime}(z)=1+2 a_{2} z+3 a_{3} z^{2}+\cdots \quad \text { and } \\
& g^{\prime}(w)=1-2 a_{2} w+3\left(2 a_{2}-a_{3}\right) w^{2}-\cdots,
\end{aligned}
$$


it follows from (2.12)-(2.15) that

$$
\begin{aligned}
& (\lambda+\mu) a_{2}=\frac{1}{2} B_{1} p_{1}, \\
& (2 \lambda+\mu) a_{3}+\frac{(\mu-1)(2 \lambda+\mu)}{2} a_{2}^{2}=\frac{1}{2} B_{1}\left(p_{2}-\frac{1}{2} p_{1}^{2}\right)+\frac{1}{4} B_{2} p_{1}^{2}, \\
& -(\lambda+\mu) a_{2}=\frac{1}{2} B_{1} q_{1}
\end{aligned}
$$

and

$$
-(2 \lambda+\mu) a_{3}+\frac{(3+\mu)(2 \lambda+\mu)}{2} a_{2}^{2}=\frac{1}{2} B_{1}\left(q_{2}-\frac{1}{2} q_{1}^{2}\right)+\frac{1}{4} B_{2} q_{1}^{2} .
$$

From (2.16) and (2.18), we get

$$
p_{1}=-q_{1}
$$

and

$$
8(\lambda+\mu)^{2} a_{2}^{2}=B_{1}^{2}\left(p_{1}^{2}+q_{1}^{2}\right) .
$$

Also, from (2.17) and (2.19), we obtain

$$
(1+\mu)(2 \lambda+\mu) a_{2}^{2}=\frac{1}{2} B_{1}\left(p_{2}+q_{2}\right)+\frac{1}{4}\left(B_{2}-B_{1}\right)\left(p_{1}^{2}+q_{1}^{2}\right),
$$

or

$$
a_{2}^{2}=\frac{2 B_{1}\left(p_{2}+q_{2}\right)+\left(B_{2}-B_{1}\right)\left(p_{1}^{2}+q_{1}^{2}\right)}{4(1+\mu)(2 \lambda+\mu)} .
$$

Since $\left|p_{i}\right| \leq 2$ and $\left|q_{i}\right| \leq 2(i=1,2)$, it follows from (2.21) and (2.22) that

$$
\left|a_{2}\right| \leq \frac{B_{1}}{\lambda+\mu}
$$

and

$$
\left|a_{2}\right| \leq \sqrt{\frac{2\left(B_{1}+\left|B_{2}-B_{1}\right|\right)}{(1+\mu)(2 \lambda+\mu)}},
$$

which yields the desired estimate on $\left|a_{2}\right|$ as asserted in (2.5).

Next, in order to find the bound on $\left|a_{3}\right|$, by subtracting (2.19) from (2.17), we get

$$
2(2 \lambda+\mu)\left(a_{3}-a_{2}^{2}\right)=\frac{1}{2} B_{1}\left(p_{2}-q_{2}\right)+\frac{1}{4}\left(B_{2}-B_{1}\right)\left(p_{1}^{2}-q_{1}^{2}\right) .
$$

Using (2.20) and (2.21) in (2.25), we have

$$
a_{3}=\frac{1}{4(2 \lambda+\mu)} B_{1}\left(p_{2}-q_{2}\right)+\frac{1}{4(\lambda+\mu)^{2}} B_{1}^{2} p_{1}^{2},
$$


which evidently yields

$$
\left|a_{3}\right| \leq \frac{B_{1}}{2 \lambda+\mu}+\frac{B_{1}^{2}}{(\lambda+\mu)^{2}} .
$$

On the other hand, by using (2.20) and (2.22) in (2.25), we obtain

$$
a_{3}=\frac{B_{1}\left[(\mu+3) p_{2}+(1-\mu) q_{2}\right]+\left(B_{2}-B_{1}\right)\left(p_{1}^{2}+q_{1}^{2}\right)}{4(1+\mu)(2 \lambda+\mu)},
$$

and applying $\left|p_{i}\right| \leq 2$ and $\left|q_{i}\right| \leq 2(i=1,2)$ for (2.27), we get

$$
\left|a_{3}\right| \leq \frac{B_{1}}{2(2 \lambda+\mu)}\left[\frac{\mu+3}{1+\mu}+\frac{|1-\mu|}{1+\mu}\right]+\frac{2\left|B_{2}-B_{1}\right|}{(1+\mu)(2 \lambda+\mu)} .
$$

Now, we consider the bounds on $\left|a_{3}\right|$ according to $\mu$.

Case 1. If $0 \leq \mu<1$, then from (2.28)

$$
\left|a_{3}\right| \leq \frac{2\left(B_{1}+\left|B_{2}-B_{1}\right|\right)}{(1+\mu)(2 \lambda+\mu)} .
$$

Case 2. If $\mu \geq 1$, then from (2.28)

$$
\left|a_{3}\right| \leq \frac{B_{1}}{2 \lambda+\mu}+\frac{2\left|B_{2}-B_{1}\right|}{(1+\mu)(2 \lambda+\mu)} .
$$

Thus, from (2.26), (2.29) and (2.30), we obtain the desired estimate on $\left|a_{3}\right|$ given in (2.6). This completes the proof of Theorem 2.1.

Putting $\mu=1$ and $\lambda=\mu=1$ in Theorem 2.1, we respectively get the following Corollaries 2.1 and 2.2.

Corollary 2.1 If $f \in H_{\sigma}(\lambda, \varphi)(\lambda \geq 1)$, then

$$
\left|a_{2}\right| \leq \min \left\{\frac{B_{1}}{\lambda+1}, \sqrt{\frac{B_{1}+\left|B_{2}-B_{1}\right|}{2 \lambda+1}}\right\}
$$

and

$$
\left|a_{3}\right| \leq \begin{cases}\min \left\{\frac{B_{1}}{2 \lambda+1}+\frac{B_{1}^{2}}{(\lambda+1)^{2}}, \frac{B_{1}+\left|B_{2}-B_{1}\right|}{2 \lambda+1}\right\}, & 0 \leq \mu<1 \\ \frac{B_{1}+\left|B_{2}-B_{1}\right|}{2 \lambda+1}, & \mu \geq 1\end{cases}
$$

Corollary 2.2 Iff $\in H_{\sigma}(\varphi)$, then

$$
\left|a_{2}\right| \leq \min \left\{\frac{B_{1}}{2}, \sqrt{\frac{B_{1}+\left|B_{2}-B_{1}\right|}{3}}\right\}
$$

and

$$
\left|a_{3}\right| \leq \begin{cases}\min \left\{\frac{B_{1}}{3}+\frac{B_{1}^{2}}{4}, \frac{B_{1}+\left|B_{2}-B_{1}\right|}{3}\right\}, & 0 \leq \mu<1, \\ \frac{B_{1}+\left|B_{2}-B_{1}\right|}{3}, & \mu \geq 1\end{cases}
$$


Remark 2.1 The estimates of the coefficients $\left|a_{2}\right|$ and $\left|a_{3}\right|$ of Corollaries 2.1 and 2.2 are the improvement of the estimates obtained in [14, Theorem 2.1] and [13, Theorem 2.1], respectively.

Remark 2.2 If we set

$$
\varphi(z)=\frac{1+(1-2 \beta) z}{1-z}=1+2(1-\beta) z+2(1-\beta) z^{2}+\cdots \quad(0 \leq \beta<1)
$$

in Corollaries 2.1 and 2.2, the results obtained improve the results in [11, Theorem 3.2, inequalities (3.3) and (3.4)] and [10, Theorem 2, inequality (3.3)], respectively.

Definition 2.2 Let $\gamma \in C^{*}=C \backslash\{0\}, \lambda \geq 0$ and $\mu \geq 0$. A function $f \in \sigma$ given by (1.1) is said to be in the class $M_{\sigma}^{\gamma}(\lambda, \mu, \varphi)$, if the following subordinations hold:

$$
1+\frac{1}{\gamma}\left(\frac{z f^{\prime}(z)+(2 \lambda \mu+\lambda-\mu) z^{2} f^{\prime \prime}(z)+\lambda \mu z^{3} f^{\prime \prime \prime}(z)}{(1-\lambda+\mu) f(z)+(\lambda-\mu) z f^{\prime}(z)+\lambda \mu z^{2} f^{\prime \prime}(z)}-1\right) \prec \varphi(z)
$$

and

$$
1+\frac{1}{\gamma}\left(\frac{w g^{\prime}(w)+(2 \lambda \mu+\lambda-\mu) w^{2} g^{\prime \prime}(w)+\lambda \mu w^{3} g^{\prime \prime \prime}(w)}{(1-\lambda+\mu) g(w)+(\lambda-\mu) w g^{\prime}(w)+\lambda \mu w^{2} g^{\prime \prime}(w)}-1\right) \prec \varphi(w),
$$

where the function $g$ is defined by (2.4).

We note that, by choosing appropriate values for $\lambda, \mu, \gamma$ and $\varphi$, the class $M_{\sigma}^{\gamma}(\lambda, \mu, \varphi)$ reduces to several earlier known classes.

(1) $M_{\sigma}^{\gamma}(\lambda, 0, \varphi)=N_{\sigma, \gamma}^{\lambda}(\varphi)\left(\lambda \geq 0, \gamma \in C^{*}\right)$ (see Kumar et al. [14, Definition 2.2]);

(2) $M_{\sigma}^{1}\left(0,0, \frac{1+(1-2 \beta) z}{1-z}\right)=S_{\sigma}^{*}(\beta)(0 \leq \beta<1)$ (see Brannan and Taha [6, Definition 3.1]);

(3) $M_{\sigma}^{1}\left(1,0, \frac{1+(1-2 \beta) z}{1-z}\right)=K_{\sigma}(\beta)(0 \leq \beta<1)$ (see Brannan and Taha [6, Definition 4.1]);

(4) $M_{\sigma}^{1}\left(0,0,\left(\frac{1+z}{1-z}\right)^{\alpha}\right)=S_{\sigma}^{*}(\alpha)(0<\alpha \leq 1)$ (see Taha [7]).

For functions in the class $M_{\sigma}^{\gamma}(\lambda, \mu, \varphi)$, the following estimates are derived.

Theorem 2.2 Let $\gamma \in C^{*}, \lambda \geq 0$ and $\mu \geq 0$. Iff $\in M_{\sigma}^{\gamma}(\lambda, \mu, \varphi)$, then

$$
\left|a_{2}\right| \leq \frac{|\gamma| B_{1} \sqrt{B_{1}}}{\sqrt{\left|\left[2(6 \lambda \mu+2 \lambda-2 \mu+1)-(2 \lambda \mu+\lambda-\mu+1)^{2}\right] B_{1}^{2} \gamma+2(2 \lambda \mu+\lambda-\mu+1)^{2}\left(B_{1}-B_{2}\right)\right|}}
$$

and

$$
\left|a_{3}\right| \leq \frac{|\gamma|\left(B_{1}+\left|B_{2}-B_{1}\right|\right)}{\left|2(6 \lambda \mu+2 \lambda-2 \mu+1)-(2 \lambda \mu+\lambda-\mu+1)^{2}\right|} .
$$

Proof If $f \in M_{\sigma}^{\gamma}(\lambda, \mu, \varphi)$, then there are analytic functions $u, v: D \rightarrow D$, with $u(0)=v(0)=$ 0 , satisfying

$$
1+\frac{1}{\gamma}\left(\frac{z f^{\prime}(z)+(2 \lambda \mu+\lambda-\mu) z^{2} f^{\prime \prime}(z)+\lambda \mu z^{3} f^{\prime \prime \prime}(z)}{(1-\lambda+\mu) f(z)+(\lambda-\mu) z f^{\prime}(z)+\lambda \mu z^{2} f^{\prime \prime}(z)}-1\right)=\varphi(u(z))
$$


and

$$
1+\frac{1}{\gamma}\left(\frac{w g^{\prime}(w)+(2 \lambda \mu+\lambda-\mu) w^{2} g^{\prime \prime}(w)+\lambda \mu w^{3} g^{\prime \prime \prime}(w)}{(1-\lambda+\mu) g(w)+(\lambda-\mu) w g^{\prime}(w)+\lambda \mu w^{2} g^{\prime \prime}(w)}-1\right)=\varphi(v(w))
$$

Let $p$ and $q$ be defined as in (2.8), then it is clear from (2.33), (2.34), (2.9) and (2.10) that

$$
\begin{aligned}
1+ & \frac{1}{\gamma}\left(\frac{z f^{\prime}(z)+(2 \lambda \mu+\lambda-\mu) z^{2} f^{\prime \prime}(z)+\lambda \mu z^{3} f^{\prime \prime \prime}(z)}{(1-\lambda+\mu) f(z)+(\lambda-\mu) z f^{\prime}(z)+\lambda \mu z^{2} f^{\prime \prime}(z)}-1\right) \\
& =\varphi\left(\frac{p(z)-1}{p(z)+1}\right)
\end{aligned}
$$

and

$$
\begin{aligned}
1+ & \frac{1}{\gamma}\left(\frac{w g^{\prime}(w)+(2 \lambda \mu+\lambda-\mu) w^{2} g^{\prime \prime}(w)+\lambda \mu w^{3} g^{\prime \prime \prime}(w)}{(1-\lambda+\mu) g(w)+(\lambda-\mu) w g^{\prime}(w)+\lambda \mu w^{2} g^{\prime \prime}(w)}-1\right) \\
& =\varphi\left(\frac{q(w)-1}{q(w)+1}\right) .
\end{aligned}
$$

It follows from (2.35), (2.36), (2.14) and (2.15) that

$$
\begin{aligned}
& (2 \lambda \mu+\lambda-\mu+1) a_{2}=\frac{1}{2} B_{1} p_{1} \gamma \\
& -(2 \lambda \mu+\lambda-\mu+1)^{2} a_{2}^{2}+2(6 \lambda \mu+2 \lambda-2 \mu+1) a_{3} \\
& \quad=\gamma\left[\frac{1}{2} B_{1}\left(p_{2}-\frac{1}{2} p_{1}^{2}\right)+\frac{1}{4} B_{2} p_{1}^{2}\right], \\
& -(2 \lambda \mu+\lambda-\mu+1) a_{2}=\frac{1}{2} B_{1} q_{1} \gamma
\end{aligned}
$$

and

$$
\begin{aligned}
& {\left[4(6 \lambda \mu+2 \lambda-2 \mu+1)-(2 \lambda \mu+\lambda-\mu+1)^{2}\right] a_{2}^{2}-2(6 \lambda \mu+2 \lambda-2 \mu+1) a_{3}} \\
& \quad=\gamma\left[\frac{1}{2} B_{1}\left(q_{2}-\frac{1}{2} q_{1}^{2}\right)+\frac{1}{4} B_{2} q_{1}^{2}\right] .
\end{aligned}
$$

Equations (2.37) and (2.39) yield

$$
p_{1}=-q_{1}
$$

and

$$
8(2 \lambda \mu+\lambda-\mu+1)^{2} a_{2}^{2}=B_{1}^{2} \gamma^{2}\left(p_{1}^{2}+q_{1}^{2}\right) .
$$

From (2.38), (2.40), (2.41) and (2.42), it follows that

$$
a_{2}^{2}=\frac{\gamma^{2} B_{1}^{3}\left(p_{2}+q_{2}\right)}{4\left[\left(2(6 \lambda \mu+2 \lambda-2 \mu+1)-(2 \lambda \mu+\lambda-\mu+1)^{2}\right) B_{1}^{2} \gamma+(2 \lambda \mu+\lambda-\mu+1)^{2}\left(B_{1}-B_{2}\right)\right]}
$$

which yields the desired estimate on $\left|a_{2}\right|$ as described in (2.31). 
Similarly, it can be obtained from (2.38), (2.40) and (2.41) that

$$
\begin{aligned}
a_{3}= & \frac{\gamma B_{1}\left[p_{2}\left(4(6 \lambda \mu+2 \lambda-2 \mu+1)-(2 \lambda \mu+\lambda-\mu+1)^{2}\right)+q_{2}(2 \lambda \mu+\lambda-\mu+1)^{2}\right]}{8\left[2(6 \lambda \mu+2 \lambda-2 \mu+1)-(2 \lambda \mu+\lambda-\mu+1)^{2}\right](6 \lambda \mu+2 \lambda-2 \mu+1)} \\
& +\frac{2 \gamma\left(B_{2}-B_{1}\right)(6 \lambda \mu+2 \lambda-2 \mu+1) p_{1}^{2}}{8\left[2(6 \lambda \mu+2 \lambda-2 \mu+1)-(2 \lambda \mu+\lambda-\mu+1)^{2}\right](6 \lambda \mu+2 \lambda-2 \mu+1)}
\end{aligned}
$$

which easily leads to the desired estimate (2.32) on $\left|a_{3}\right|$.

Taking $\mu=0$ in Theorem 2.2, we obtain the following corollary.

Corollary 2.3 [14, Theorem 2.3] If $\in \in N_{\sigma, \gamma}^{\lambda}(\varphi)$, then

$$
\left|a_{2}\right| \leq \frac{|\gamma| B_{1} \sqrt{B_{1}}}{\sqrt{\left|\left(1+2 \lambda-\lambda^{2}\right) B_{1}^{2} \gamma+(1+\lambda)^{2}\left(B_{1}-B_{2}\right)\right|}} \quad \text { and } \quad\left|a_{3}\right| \leq \frac{|\gamma|\left(B_{1}+\left|B_{2}-B_{1}\right|\right)}{\left|1+2 \lambda-\lambda^{2}\right|} \text {. }
$$

Further, for $\gamma=1$, putting $\lambda=0$ and $\lambda=1$ in Corollary 2.3, respectively, we have the following Corollaries 2.4 and 2.5.

Corollary 2.4 [13, Corollary 2.1] Iff $\in M_{\sigma}^{1}(0,0, \varphi)=S T_{\sigma}(\varphi)$, then

$$
\left|a_{2}\right| \leq \frac{B_{1} \sqrt{B_{1}}}{\sqrt{\left|B_{1}^{2}+B_{1}-B_{2}\right|}} \text { and }\left|a_{3}\right| \leq B_{1}+\left|B_{2}-B_{1}\right|
$$

Corollary 2.5 [13, Corollary 2.2] Iff $\in M_{\sigma}^{1}(1,0, \varphi)=C V_{\sigma}(\varphi)$, then

$$
\left|a_{2}\right| \leq \frac{B_{1} \sqrt{B_{1}}}{\sqrt{2\left|B_{1}^{2}+2 B_{1}-2 B_{2}\right|}} \quad \text { and } \quad\left|a_{3}\right| \leq \frac{1}{2}\left(B_{1}+\left|B_{2}-B_{1}\right|\right)
$$

Remark 2.3 If we set

$$
\varphi(z)=\left(\frac{1+z}{1-z}\right)^{\alpha}=1+2 \alpha z+2 \alpha^{2} z^{2}+\cdots \quad(0<\alpha \leq 1)
$$

and

$$
\varphi(z)=\frac{1+(1-2 \beta) z}{1-z}=1+2(1-\beta) z+2(1-\beta) z^{2}+\cdots \quad(0 \leq \beta<1)
$$

in Corollaries 2.4 and 2.5, we obtain the results of Brannan and Taha [6, Theorems 2.1, 3.1 and 4.1, respectively]. 


\section{Acknowledgements}

The present investigation was partly supported by the Natural Science Foundation of People's Republic of China under Grant 11271045, the Higher School Doctoral Foundation of People's Republic of China under Grant 20100003110004 and the Natural Science Foundation of Inner Mongolia of People's Republic of China under Grant 2010MS0117. The authors are thankful to the referees for their careful reading and making some helpful comments which have essentially improved the presentation of this paper.

Received: 9 November 2012 Accepted: 14 June 2013 Published: 8 July 2013

\section{References}

1. Duren, PL: Univalent Functions. Grundlehren der Mathematischen Wissenschaften, Band 259. Springer, Berlin (1983)

2. Srivastava, HM, Owa, S (eds.): Current Topics in Analytic Function Theory. World Scientific, Singapore (1992)

3. Lewin, M: On a coefficient problem for bi-univalent functions. Proc. Am. Math. Soc. 18, 63-68 (1967)

4. Brannan, DA, Clunie, JG (eds.): Aspects of contemporary complex analysis. In: Proceedings of the NATO Advanced Study Institute held at the University of Durham, Durham, July 1-20, 1979. Academic Press, London (1980)

5. Netanyahu, E: The minimal distance of the image boundary from the origin and the second coefficient of a univalent function in $|z|<1$. Arch. Ration. Mech. Anal. 32, 100-112 (1969)

6. Brannan, DA, Taha, TS: On some classes of bi-univalent functions. In: Mazhar, SM, Hamoui, A, Faour, NS (eds.) Mathematical Analysis and Its Applications, Kuwait, February 18-21, 1985. KFAS Proceedings Series, vol. 3, pp. 53-60. Pergamon, Oxford, (1988) see also Stud. Univ. Babes-Bolyai, Math. 31(2), $70-77$ (1986)

7. Taha, TS: Topics in Univalent Function Theory. Ph.D. thesis, University of London (1981)

8. Brannan, DA, Clunie, J, Kirwan, WE: Coefficient estimates for a class of star-like functions. Can. J. Math. 22, 476-485 (1970)

9. Ma, WC, Minda, D: A unified treatment of some special classes of univalent functions. In: Proceedings of the Conference on Complex Analysis, Tianjin, 1992. Conf. Proc. Lecture Notes Anal., vol. I, pp. 157-169. International Press, Cambridge (1994)

10. Srivastava, HM, Mishra, AK, Gochhayat, P: Certain subclasses of analytic and bi-univalent functions. Appl. Math. Lett. 23, 1188-1192 (2010)

11. Frasin, BA, Aouf, MK: New subclasses of bi-univalent functions. Appl. Math. Lett. 24, 1569-1573 (2011)

12. Caglar, M, Orhan, H, Yagmur, N: Coefficient bounds for new subclasses of bi-univalent functions. Filomat (2013, in press)

13. Ali, RM, Lee, SK, Ravichandran, V, Supramaniam, S: Coefficient estimates for bi-univalent Ma-Minda starlike and convex functions. Appl. Math. Lett. 25, 344-351 (2012)

14. Kumar, SS, Kumar, V, Ravichandran, V: Estimates for the initial coefficients of bi-univalent functions. Preprint

15. Srivastava, HM, Bulut, S, Cağlar, M, Yağmur, N: Coefficient estimates for a general subclass of analytic and bi-univalent functions. Filomat 27, 831-842 (2013)

16. $\mathrm{Xu}, \mathrm{Q}-\mathrm{H}, \mathrm{XiaO}, \mathrm{H}-\mathrm{G}$, Srivastava, HM: A certain general subclass of analytic and bi-univalent functions and associated coefficient estimate problems. Appl. Math. Comput. 218, 11461-11465 (2012)

17. Xu, Q-H, Gui, Y-C, Srivastava, HM: Coefficient estimates for a certain subclass of analytic and bi-univalent functions. Appl. Math. Lett. 25, 990-994 (2012)

doi:10.1186/1029-242X-2013-317

Cite this article as: Tang et al.: Coefficient estimates for new subclasses of Ma-Minda bi-univalent functions. Journal of Inequalities and Applications 2013 2013:317.

\section{Submit your manuscript to a SpringerOpen ${ }^{\circ}$ journal and benefit from:}

- Convenient online submission

- Rigorous peer review

- Immediate publication on acceptance

- Open access: articles freely available online

- High visibility within the field

- Retaining the copyright to your article 